\title{
Diels-Alder Reactions of Acyclic 2-Azadienes: A Semiempirical Molecular Orbital Study
}

\author{
Teresa M. V. D. Pinho e Melo, ${ }^{\dagger}$ Rui Fausto, ${ }^{\ddagger}$ and António M. d’A. Rocha Gonsalves*,\$ \\ Departamento de Química, Universidade de Coimbra, P-3049 Coimbra, Portugal \\ Thomas L. Gilchrist" \\ Chemistry Department, University of Liverpool, Liverpool, L69 7ZD, U.K.
}

Received J anuary 20, 1998

\begin{abstract}
Molecular orbital calculations (AM1) have been performed to obtain the frontier orbitals' (HOMO and LUMO) energies and polarization of a series of acyclic 2-azadienes. The results are used to rationalize the reactivity of the compounds studied with both electron-rich and electron-deficient dienophiles as well as the observed regioselectivity of the corresponding Diels-Alder reactions.
\end{abstract}

\section{Introduction}

The Diels-Alder reaction of acyclic 2-azadienes is a useful method for the preparation of pyridines, dihydropyridines, and tetrahydropyridines. ${ }^{1}$ In the past decade, some new methods of generating 2-azadienes have been found which have increased the scope of this approach. The great majority of 2-azadienes studied are substituted with strongly electron-donating groups, and they participate in the normal Diels-Alder reaction with electrondeficient dienophiles.

We have recently drawn our attention to the study of 2-azadienes bearing one or two electron-withdrawing groups (1a-1h). The thiazolidines 2, prepared from

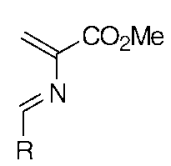

1

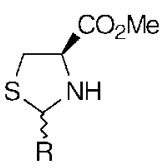

2

$$
\begin{aligned}
& \text { a: } \mathrm{R}=\mathrm{Ph} \\
& \text { b: } \mathrm{R}=4-\mathrm{Me}_{2} \mathrm{NC}_{6} \mathrm{H}_{4} \\
& \text { c: } \mathrm{R}=4-\mathrm{NO}_{2} \mathrm{C}_{6} \mathrm{H}_{4} \\
& \text { d: } \mathrm{R}=4-\mathrm{pyridyl} \\
& \text { e: } \mathrm{R}=\mathrm{COPh} \\
& \text { f: } \mathrm{R}=\mathrm{CO}_{2} \mathrm{Et} \\
& \text { g: } \mathrm{R}=\mathrm{H} \\
& \text { h: } \mathrm{R}=\mathrm{Bu}^{t}
\end{aligned}
$$

cysteine methyl ester and aldehydes, react with silver carbonate and DBU generating the corresponding azadienes. ${ }^{2}$ The Diels-Alder reaction of these azadienes with a range of dienophiles has been studied, and adducts have been isolated in all cases except when azadienes $\mathbf{l g}$ and $\mathbf{l h}$ were used. ${ }^{2}$

\footnotetext{
† E-mail: teresamelo@gemini.ci.uc.pt.

‡E-mail: rfausto@gemini.ci.uc.pt.

$\S$ E-mail: arg@gemini.ci.uc.pt.

"E-mail: tlg57@iverpool.ac.uk.

(1) (a) Boger, D. L.; Weinreb, S. M. Organic Chemistry A Series of Monographs. In Hetero Diels-Alder Methodology in Organic Syntheses; Academic Press: San Diego, 1987. (b). Boger, D. L. In Comprehensive Organic Synthesis; Trost, B. M., Fleming, I., Rds.; Pergamon Press: Oxford, 1991; Vol. 5, Chapter 4.3. (c) Barluenga, J .; Tomas M. Adv. Heterocycl. Chem. 1993, 57, 1-80. (d) Boger, D. L. J . Heterocycl. Chem. 1996, 33, 1519-1531.

(2) (a) Gilchrist, T. L.; Rocha Gonsalves, A. M. d'A.; Pinho e Melo,

\begin{tabular}{|c|c|c|}
\hline azadiene & dienophile & products $(\%)$ \\
\hline $1 \mathbf{a}$ & $\mathrm{COMe}$ & $4 \mathbf{a}(20) 3 \mathbf{a}(5) 5 \mathbf{a}(51)$ \\
\hline $1 \mathrm{~b}$ & $\mathrm{DMe}$ & $4 b(9) 5 c(43)$ \\
\hline $1 \mathbf{a}$ & $\mathrm{O}_{2} \mathrm{Me}$ & $\mathbf{3 a}(15) \mathbf{5} \mathbf{b}(4) 7 \mathbf{a}^{\mathbf{a}}(7)$ \\
\hline $1 \mathbf{a}$ & CN & $3 \mathbf{b}(7)$ \\
\hline $1 \mathbf{a}$ & $\mathrm{EtO}_{2} \mathrm{C}^{-}$ & $7 \mathrm{~b}(15)$ \\
\hline $1 \mathbf{a}$ & $\equiv-\mathrm{CO}_{2} \mathrm{Et}$ & $6 \mathbf{a}(7)$ \\
\hline $1 \mathrm{a}$ & $\mathrm{EtO}_{2} \mathrm{C}=\mathrm{CO}_{2} \mathrm{Et}$ & $6 b \quad 7 b^{b}$ \\
\hline
\end{tabular}
T. M. V. D. Tetrahedron Lett. 1993, 34, 6945-6946. (b) Gilchrist, T. L.; Rocha Gonsalves, A. M. d'A.; Pinho e Melo, T. M. V. D. Tetrahedron 1994, 50, 13709-13724. (c) Gilchrist, T. L.; Rocha Gonsalves, A. M. d'A.; Pinho e Melo, T. M. V. D. Pure Appl. Chem. 1996, 68, 859862.
}

Table 1. Reactions of Azadienes $1 \mathrm{a}$ and $1 \mathrm{~b}$ with Electron-Deficient Dienophiles 2

Azadiene 1a, bearing one electron-withdrawing substituent $\left(\mathrm{CO}_{2} \mathrm{Me}\right)$, was expected to be electron-deficient and, therefore, more likely to react with electron-rich dienophiles in the inverse Diels-Alder reaction. However, la participates in the Diels-Alder reaction with both electron-rich (inverse Diels-Alder reaction) and electron-deficient (normal Diels-Alder reaction) dienophiles, a reactivity unusual for dienes. ${ }^{1}$ On the other hand, azadiene $\mathbf{1 b}$ only takes part in the normal DielsAlder reaction while azadienes $\mathbf{1 c}$ - $\mathbf{l f}$ participate in the inverse cycl oaddition reaction. ${ }^{2}$

Normal Diels-Alder reaction of azadiene la with but3-en-2-one leads to the formation of three compounds in an overall yield of $76 \%$ (Table 1). Diels-Alder reaction of la with other electron-deficient dienophiles leads to the corresponding cycloadducts in moderate to low yield. These results allow us to conclude that the normal electron demand Diels-Alder reaction of azadiene 1a needs very activated dienophiles in order to be efficient. 
<smiles>[X]C1CC=C(C(O[Na])O[Na])N[C@H]1c1ccccc1</smiles><smiles>COC(C)=C1CC[C@@H](OC)C(P)N1</smiles>

4 a: $\mathrm{R}=\mathrm{Ph}$

3 a: $X=C O M e$ 3 b: $\mathrm{X}=\mathrm{CO}_{2} \mathrm{Me}$ $3 \mathrm{c}: \mathrm{X}=\mathrm{CN}$ 4 b: $\mathrm{R}=4-\mathrm{Me}_{2} \mathrm{NC}_{6} \mathrm{H}_{4}$<smiles>[X]C1=C(P)NC(C(C)=O)CC1</smiles>

5 a: $X=$ COMe, $R=P h$ 5 b: $X=\mathrm{CO}_{2} \mathrm{Me}, \mathrm{R}=\mathrm{Ph}$ 5 c: $\mathrm{X}=\mathrm{CO}_{2} \mathrm{Me}$, $\mathrm{R}=4-\mathrm{Me}_{2} \mathrm{NC}_{6} \mathrm{H}_{4}$<smiles>[Y]C1=C(C(=O)OCC)C(c2ccccc2)NC(C(C)=O)=C1</smiles>

6 a: $Y=H$ $6 \mathrm{~b}: \mathrm{X}=\mathrm{CO}_{2} \mathrm{Et}$<smiles>[X]c1cc(OC(C)=O)nc(-c2ccccc2)c1[X]</smiles>

7 a: $\mathrm{X}=\mathrm{CO}_{2} \mathrm{Me}, \mathrm{Y}=\mathrm{H}$

7 b: $X=Y=\mathrm{CO}_{2} \mathrm{Et}$

The same conclusion can be drawn for azadiene $\mathbf{l b}$, which also reacts with but-3-en-2-one giving the corresponding products in $52 \%$ yield.

None of the azadienes studied react with ethyl vinyl ether because it is necessary to use more activated electron-rich dienophiles (e.g., enamines) to observe the inverse Diels-Alder reaction with azadienes $\mathbf{1 a}$ and $\mathbf{1 c}-$ If (Table 2).

Table 2. Cycloaddition Reactions of Azadiene 1a and 1c-If with $\mathrm{N}$-Cyclohex-1-enylpyrrolidine (A) and $\mathbf{N}$-cyclopenten-1-ylpyrrolidine (B) ${ }^{\mathbf{2}}$

\begin{tabular}{ccc}
\hline azadiene & dienophile & products (\%) \\
\hline $\mathbf{1 a}$ & $\mathbf{A}$ & $\mathbf{8}(37), \mathbf{9 a}(20)$ \\
$\mathbf{1 c}$ & $\mathbf{A}$ & $\mathbf{1 0}(53), \mathbf{1 1 a}(20)$ \\
$\mathbf{1 d}$ & $\mathbf{A}$ & $\mathbf{1 1 b}(54)$ \\
$\mathbf{1 e}$ & $\mathbf{A}$ & $\mathbf{1 2 a}(35), \mathbf{1 1 c}(14)$ \\
$\mathbf{1 f}$ & $\mathbf{A}$ & $\mathbf{1 2}(26), \mathbf{1 1 d}(26)$ \\
$\mathbf{1 a}$ & $\mathbf{B}$ & $\mathbf{9 b}(35)$ \\
$\mathbf{1 c}$ & $\mathbf{B}$ & $\mathbf{1 1 e}(53)$ \\
$\mathbf{1 e}$ & $\mathbf{B}$ & $\mathbf{1 1 f}(27)$ \\
$\mathbf{1 f}$ & $\mathbf{B}$ & $\mathbf{1 1 g}(51)$
\end{tabular}

In all cases, the cycloaddition reactions show high regioselectivity but no endo/exo selectivity.

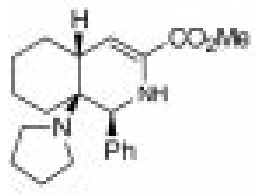

8

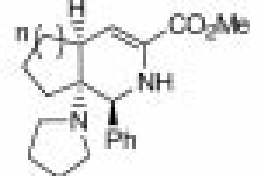

9 a: $n=2$ 9 ben $=1$

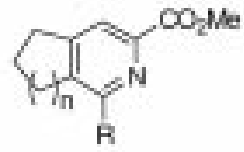

11 a: $\mathrm{A}=\mathrm{C}_{0}-\mathrm{NOO}_{2}-4, \mathrm{n}=2$

11 b: $R=4$-pyridyl, $n=2$

$11 \mathrm{c}: \mathrm{A}=\mathrm{COPh}, \mathrm{n}=2$

$11 \mathrm{~d}: \mathrm{R}=\mathrm{CO}_{2} \mathrm{Et}, \mathrm{n}=2$

$11 \mathrm{e:} \mathrm{A}=\mathrm{C}_{0} \mathrm{H}_{\mathrm{NO}}-4, n=1$

$11 \mathrm{f:}: \mathrm{A}=\mathrm{COPh}, \mathrm{n}=1$

$11 \mathrm{~g}: \mathrm{A}=\mathrm{CO}_{2} \mathrm{Et}, \mathrm{n}=1$

The above series of reactions provide a very convenient route to new tetrahydropyridines, dihydropyridines, and pyridines bearing a variety of substituents, and the understanding of the substituent effects present in the reactant molecules on their specific reactivity appears to be of fundamental importance to broaden the scope of this method of synthesis. In particular, a judicious choice of structural modifications to be made on both reactants (diene and dienophile) that is required to optimize this procedure can be greatly hel ped by having available the necessary information on their electron charge distributions, as provided by quantum mechanics theoretical methods. However, the number of theoretical studies previously undertaken on the kind of systems considered here is very low. 3,4

The Diels-Alder reaction of 2-aza-1,3-butadiene with some ethylene and acetylene derivatives has been studied previously by Hartree-Fock SCF/MO ab initio calculations using the $3-21 \mathrm{G}$ basis set. ${ }^{4}$ The calculations showed that the transition-state structures of 2-aza-1,3-butadiene and butadiene involved in the Diels-Alder reaction are similar. The $\mathrm{CH} \rightarrow \mathrm{N}$ substitution in the diene enhances its electrophilicity and lowers the energy of the rel evant MOs, making the heterodiene less reactive than 1,3butadiene toward electron-deficient dienophiles. Substitution of the azadiene with el ectron-withdrawing groups makes this species still more el ectron-deficient, favoring the inverse Diels-Alder reaction with electron-rich dienophiles or with strained dienophiles. On the other hand, substitution with electron-donating groups makes the azadiene more ready to participate in the normal Diels-Alder reaction with conventional dienophiles. The HOMO of 2-azadiene was predicted to be slightly polarized with C-1 and C-4 having approximately the same coefficients. The effect of the $\mathrm{CH} \rightarrow \mathrm{N}$ substitution on the LUMO is more pronounced, and the $\mathrm{C}-1$ coefficient becomes considerably larger than that of C-4. The predicted frontier orbitals' polarization means that C-1 is more electrophilic than C-4 (C-1 coefficient larger in the LUMO), while C-4 is slightly more nucleophilic than C-1 (C-4 coefficient slightly larger in the HOMO). The 3-21G calculations also point to a similar regioselectivity of substituted 2-aza-1,3-butadiene and substituted butadiene. Hence, the regioselectivity exhibited by 2-aza-1,3butadiene derivatives must be essentially controlled by the nature of the substituents.

Thus, despite the general progress already achieved by these previous studies, it appeared to be essential in order to rationalize the results obtained in the abovementioned experimental studies of 2-aza-1,3-butadiene derivatives (1a-1f) to undertake a series of systematic molecular orbital calculations on these systems. This article describes the results obtained.

\section{Computational Methods}

The molecular orbital calculations were carried out on a DEC ALPHA 7000 computer using either GAUSSIAN $92^{5}$ or MOPAC $6.0^{6}$ programs. Molecular geometries were fully optimized using the programs' default algorithms $s^{6,7}$ and the precise characterization of the rank of the critical point obtained, checked through calculation of the second derivative matrix. Thus, minimum energy

(3) Bachrach, S. M. Liu, M. J Org. Chem 1992, 57, 6736-6744

(4) González, J.; Houk, K. N. J . Org. Chem. 1992, 57, 3031-3037.

(5) Frish, M. J .; Trucks, G. W.; Head-Gordon, M.; Gill, P. M. W.; Wong, M. W.; Foresman, J . B.; J ohnson, B. J .; Schlegel, H. B.; Robb, M. A.; Replogle, E. S.; Gomperts, R.; Andres, J . L.; Raghavachari, K . Binkley, J . S.; Gonzalez, C.; Martin, R. L.; Fox, D. J .; Defrees, D. J .; Baker, J .; Stewart, J . J. P.; Pople, J. A. GAUSSIAN 92, revision C, Gaussian Inc: Pittsburgh, PA, 1992.

(6) Stewart, J . J . P. Program MOPAC Version 6.0, Frank J. Seiler Research Laboratory: U.S. Air Force Academy, CO 80840, 1990.

(7) Schlegel, H. B. Ph.D. Thesis, Queen's University, Kingston, Ontario, 1975 
Table 3. Semiempiric Calculations for 1,3-Butadiene and 2-Aza-1,3-butadiene Optimized Geometries (Nonplanar); Ionization Potential and Electronic Affinity of 1,3-Butadiene

\begin{tabular}{|c|c|c|c|c|c|c|}
\hline & \multicolumn{4}{|c|}{ energya $^{a}(\mathrm{eV})$} & \multicolumn{2}{|c|}{ energyb $^{b}(\mathrm{eV})$} \\
\hline & HOMO & LUMO & IP & EA & HOMO & LUMO \\
\hline AM1 & -9.35 & 0.46 & & & -9.78 & 0.27 \\
\hline PM3 & -9.51 & 0.28 & & & -9.97 & -0.01 \\
\hline MNDO & -9.38 & 0.59 & & & -9.50 & 0.77 \\
\hline MINDO/3 & -9.48 & 1.50 & & & -8.02 & 1.55 \\
\hline AM1c & -9.36 & 0.46 & & & -9.91 & 0.13 \\
\hline $3-21 G^{d}$ & -8.85 & 3.59 & & & -9.48 & 3.37 \\
\hline $\exp ^{e}$ & & & 9.2 & -0.6 & & \\
\hline
\end{tabular}

a For 1,3-butadiene. b For 2-aza-1,3-butadiene. c Planar optimized geometries. d See ref 4 . e See ref $1 b$.

conformations were used to compute molecular orbitals and evaluate charge electron distributions. The charge density calculations were undertaken with the program MOLDEN (Linux-redhat version) ${ }^{8}$ on a PC equipped with a Pentium P54CX processor, using the relevant data transferred from the previously undertaken MO calculations.

\section{Results and Discussion}

The first step in this study was the selection of the best method to estimate the frontier orbital energies of 2-azadienes, since these quantities assume particular relevance here.

HOMO and LUMO energies of 1,3-butadiene and 2-aza-1,3-butadiene using several MO semiempirical methods, namely AM $1,{ }^{9} \mathrm{PM} 3,{ }^{10} \mathrm{MNDO},{ }^{11}$ and MINDO/ $3,{ }^{12-14}$ for the optimized geometries (nonplanar) were calculated and compared with the available 3-21G ab initio data, ${ }^{3,4}$ the experimental ionization potential (IP), and the electron affinity (EA) of the unsubstituted diene. $^{1 \mathrm{~b}}$ Calculations were also made for planar optimized geometries to estimate the effect of geometry distortion from planarity (Table 3 ).

The experimental values of the ionization potential and electron affinity of butadiene ${ }^{1 \mathrm{~b}}$ indicate that the 3-21G calculations considerably overestimate the energies of both frontier orbitals (HOMO and LUMO). The MNDO values are better for butadiene but do not show the expected change in LUMO energy for 2-azadiene, while the results of AM 1 calculations were able to reproduce the decrease in both HOMO and LUMO energies upon $\mathrm{CH} \rightarrow \mathrm{N}$ substitution. Overall, the AM1 approach appears to be the best method to predict these quantities.

These results led us to select AMI as the method to apply to the study of azadienes 1a-1f. Full geometry optimization was performed throughout the main set of calculations in order to deal with true equilibrium structures, despite the fact that constraining the diene geometry to a planar conformation apparently does not

(8) Schaftenaar, G. MOLDEN, Caos/camm Center, Nijmegen, Toernooiveld, The Netherlands, 1991.

(9) Dewar, M. J . S. D.; Zxoebisch, E. G.; Healy, E. F.; Stewart, J . J P. J. Am. Chem. Soc. 1985, 107, 3902.

(10) Stewart, J .J . P. J . Cumput. Chem. 1989, 10, 209, 221.

(11) Dewar, M. J. S. D.; Thiel, W. J . Am. Chem. Soc. 1977, 99, 4899.

(12) Dewar, M.; Thiel, W. J . Am. Chem. Soc. 1977, 99, 4499.

(13) Bingham, R. C.; Dewar, M.; Lo. J . Am. Chem. Soc. 1975, 97, $1285,1294,1302,1307$

(14) (a) Houk, K. N.; Sims, J .; Duke, R. E., J r.; Strozier, R. W.; George, J. K. J . Am. Chem. Soc. 1973, 95, 7287-7301. (b) Desimoni, G.; Tacconi, G. Chem. Rev. 1975, 75, 651-692. (c) Ledwith, A.; Woods, H. J . J . Chem. Soc. 1970, 310-314.

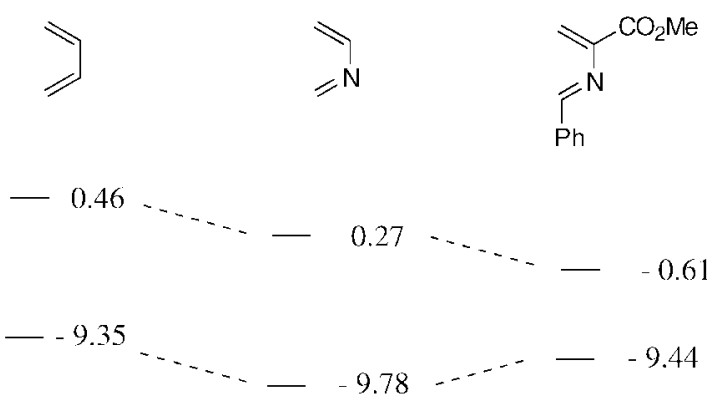

Figure 1. Frontier orbital energy for 1,3-butadiene, 2-aza1,3-butadiene, and methyl 1-phenyl-2-aza-1,3-butadiene-3carboxylate 1a (AM 1 calculations).

Table 4. AM1 Calculations for Azadienes la- If

\begin{tabular}{crc}
\hline & \multicolumn{2}{c}{ energy (eV) } \\
\cline { 2 - 3 } 2-azadiene & HOMO & LUMO \\
\hline la & -9.44 & -0.61 \\
$\mathbf{1 b}$ & -8.21 & -0.22 \\
$\mathbf{1 c}$ & -10.13 & -1.60 \\
$\mathbf{1 d}$ & -9.98 & -0.87 \\
$\mathbf{1 e}$ & -10.08 & -0.61 \\
$\mathbf{1 f}$ & -10.51 & -0.85
\end{tabular}

lead to dramatic changes in the energies of the frontier orbitals (Table 3 ).

The AM1 calculations described above were done considering the s-cis conformation of butadiene and 2-aza-1,3-butadiene. However we also performed AM 1 calculations for the compounds in the s-trans conformation, finding that in both cases the s-trans conformation is more stable than the s-cis conformation. However the difference in energy is small $(\Delta \mathrm{E} \sim 1 \mathrm{kcal} / \mathrm{mol})$. The HOMO and LUMO energy for butadiene and 2-aza-1,3butadiene in the s-trans conformation is almost identical to the one for the corresponding s-cis conformation [butadiene in s-trans conformation HOMO $-9.34 \mathrm{eV}$ and LUMO 0.45 eV; 2-aza-1,3-butadiene in s-trans conformation HOMO -9.79 and LUMO $0.24 \mathrm{eV}]$. Since the same is true for 1-monosubstituted-2-aza-1,3-butadiene-3-carboxylates we only performed the calculations of these derivatives for the s-cis conformation.

The MO semiempirical method allowed the study of more complex 2-azadiene derivatives including those not available in the traditional ab initio approach.

Reactivity of 2-Azadienes 1a-1f. Table 4 shows the frontier orbital energies of azadiene $\mathbf{1 a - 1 f}$ determined by AM 1 calculations. For azadienes $\mathbf{l e}$ and $\mathbf{1 f}$ calculations for two different conformations (differing in the relative orientation of the $\mathrm{R}$ substituent) were made, but similar results were obtained.

As referred to before, azadiene 1a has an unusual reactivity characterized by participation in both normal and inverse Diels-Alder reactions. Figure 1 shows the calculated HOMO and LUMO energy for 1,3-butadiene, 2-aza-1,3-butadiene, and azadiene 1a. It is clear from this figure that the effect of substitution is to get the HOMO and LUMO energy levels closer. Indeed, the effect of the methoxycarbonyl group is essentially to lower both energy levels, whereas the phenyl group raises the HOMO and lowers the LUMO energies.

It is well-known that butadiene reacts easily with electron-deficient dienophiles. The small energy gap between the HOMO of butadiene and of the heterodiene la explains the participation of this azadiene in the 
Table 5.

\begin{tabular}{clc}
\hline & & $\Delta \mathrm{E}(\mathrm{eV})$ \\
azadiene $^{\mathrm{a}}$ & \multicolumn{1}{c}{ dienophile } & (LUMO $\left._{\text {azadiene }}-\mathrm{HOMO} \mathrm{O}_{\text {dienophile }}\right)$ \\
\hline $\mathbf{l a}$ & $\mathbf{B}$ & 6.72 \\
$\mathbf{l a}$ & ethyl vinyl ether & 8.19 \\
$\mathbf{1 k}$ & $\mathbf{B}$ & 5.07 \\
$\mathbf{1 k}$ & ethyl vinyl ether & 6.54
\end{tabular}

a LUMO energy of the azadienes determined by AM 1 calculations. ${ }^{b}$ HOMO energy of the dienophiles estimated from the ionization potential: $\mathrm{N}$-cyclopenten-1-ylpyrrolidine, $7.33 \mathrm{eV}$; ethyl vinyl ether, $8.80 \mathrm{eV} .{ }^{14}$

normal electron demand Diels-Alder reaction. On the other hand, the LUMO energy of $\mathbf{1 a}$ is considerably lower than in both butadiene and 2-aza-1,3-butadiene, thus explaining the participation of $\mathbf{1 a}$ in the inverse DielsAlder reaction. However, the inverse cycloaddition can only occur with strongly activated dienophiles (Table 5).

The value of the HOMO energy obtained for $\mathbf{1 b}$ is $-8.21 \mathrm{eV}$, which means that this azadiene should be more reactive toward el ectron-deficient dienophiles than $\mathbf{1 a}$. The experimental results confirm the participation of $\mathbf{1 b}$ in the normal cycloaddition (Table 1). However, in this case, besides considerably increasing the HOMO energy, the substituent 4-(dimethylamino)phenyl in C-1 of azadiene $\mathbf{1 b}$ also significantly raises the LUMO energy, thus making this compound unable to react with electron-rich dienophiles.

The calculated HOMO energy of azadienes $\mathbf{1 c}-\mathbf{l f}$ is much lower than that of $\mathbf{1 a}$ and $\mathbf{1 b}$. This explains the lack of reactivity of these azadienes toward electrondeficient dienophiles (normal Diels-Alder reaction). On the other hand, the predicted LUMO energy of $\mathbf{1 c}-\mathbf{1 f}$ is lower than that of $\mathbf{1 a}$ (in the case of $\mathbf{1 e}$, nearly the same), pointing to an increased reactivity toward electron-rich dienophiles (inverse Diels-Alder reaction).

Azadiene 1c, bearing a 4-nitrophenyl group at C-1, has the lowest LUMO energy, followed by $\mathbf{l d}$ and $\mathbf{1 f}$. This indicates that the addition of one nitro group at the para position of the phenyl substituent of the azadiene has a bigger effect than the introduction of one nitrogen at the same position, or the substitution of the aryl group by one ethoxycarbonyl moiety. The LUMO energy of azadiene $\mathbf{1 d}$ and $\mathbf{l f}$ is predicted to be similar.

It can then be concluded, considering the AM 1 calculated LUMO relative energies along the series of azadienes studied, that their order of decreasing reactivity to participate in the inverse el ectron demand Diels-Alder reaction shall be the following: $\mathbf{1 c}>\mathbf{1 d} \sim \mathbf{1 f}>\mathbf{1 e} \sim \mathbf{1 a}$ $>\mathbf{l b}$.

It has been experimentally found that the inverse Diels-Alder reaction of these azadienes with $\mathrm{N}$-cyclohex1-enyl pyrrol idine leads, in general, to better yields than the reaction with $\mathrm{N}$-cyclopenten-1-pyrrolidine (Table 2). This result agrees with the fact that $\mathrm{N}$-cyclohex-1-enylpyrrolidine is a slightly more activated dienophile (higher HOMO energy ${ }^{15}$ ) and is also consistent with the theoretical interpretation of relative reactivity of the azadienes based on the AM1 data.

A particularly important experimental result that is also properly predicted by the AM 1 calculations is the higher reactivity shown by azadiene $\mathbf{1 c}$ in the inverse Diels-Alder reaction with enamines, when compared with all other azadienes studied. Unfortunately, it has

(15) Sustmann, R. Pure Appl. Chem. 1974, 46, 569-593.
Table 6. AM1 Calculations for Azadienes $\mathbf{1} \mathbf{i}-\mathbf{1 r}$

\begin{tabular}{|c|c|c|}
\hline \multirow[b]{2}{*}{ 2-azadiene } & \multicolumn{2}{|c|}{ energy (eV) } \\
\hline & HOMO & LUMO \\
\hline $\mathbf{1 i}$ & -9.41 & -0.37 \\
\hline $\mathbf{1 j}$ & -10.26 & -1.87 \\
\hline $\mathbf{l} \mathbf{k}$ & -10.45 & -2.26 \\
\hline 1) & -10.59 & -1.35 \\
\hline $1 \mathrm{~m}$ & -10.58 & -1.71 \\
\hline In & -9.50 & -0.60 \\
\hline 10 & -9.57 & -0.80 \\
\hline $1 p$ & -9.40 & -0.80 \\
\hline $1 q$ & -10.54 & -1.19 \\
\hline $1 \mathbf{r}$ & -9.70 & -1.18 \\
\hline
\end{tabular}

not yet been possible to confirm experimentally the predicted order of reactivity for the other azadienes.

After the publication of our first experimental results, ${ }^{2}$ Balsamini et al. ${ }^{16}$ reported studies on derivatives of 2-aza1,3-butadiene-3-carboxylic acids, namely, methyl 1,2diphenyl-2-aza-1,3-butadi ene-3-carboxylate 1i (Table 6).<smiles>C=C(N=C(c1ccccc1)c1ccccc1)C(C)=O</smiles>

$1 \mathrm{i}$<smiles>C=C(C)C(C)=N/C=C/c1cnnc(C(=C)CC)n1</smiles>

11

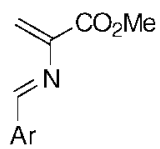

1j: $\quad \operatorname{Ar}=2,6$-dinitrophenyl 1k: $\mathrm{Ar}=2,4$-dinitrophenyl

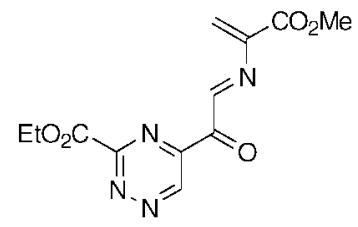

$1 \mathrm{~m}$

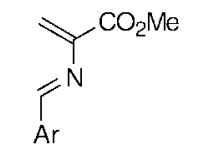

1n: $\mathrm{Ar}=2-\mathrm{F}-\mathrm{C}_{6} \mathrm{H}_{4}$

10: $\mathrm{Ar}=3-\mathrm{F}-\mathrm{C}_{6} \mathrm{H}_{4}$

1p: $\mathrm{Ar}=4-\mathrm{F} \cdot \mathrm{C}_{6} \mathrm{H}_{4}$

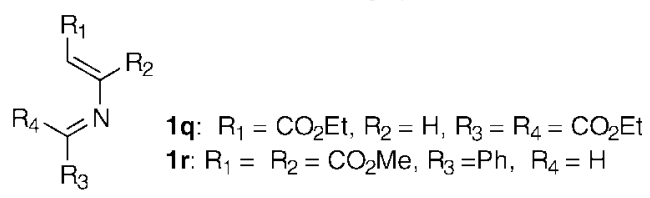

This azadiene only differs from $\mathbf{l a}$ because it has an additional phenyl group, but the observed reactivities are not the same. Azadiene 1i participates in the normal Diels-Alder reaction only with dienophiles bearing strong electron-withdrawing activating groups (e.g., dimethyl acetylenedicarboxylate, tetracyanoethylene, and 4-phenyl-1,2,4-triazoline-3,5-dione), no reaction being observed with less-activated dienophiles (e.g., methyl propiolate, methyl acrylate, and acrolein). On the other hand, no adducts could be isolated from the attempted inverse Diels-Alder reaction with $\mathrm{N}$-cyclohex-1-enylpyrrolidine. Thus, contrary to $\mathbf{l a}$, which can participate in both normal and inverse Diels-Alder reaction, azadiene $\mathbf{l i}$ was found to participate only in the normal Diels-Alder reaction and only with strongly activated dienophiles. Being aware of our work, Balsamini et al. could not

(16) Balsamini, C.; Bedini, A.; Galarini, R.; Spadoni, G.; Tarzia, G. Hamdan, M. Tetrahedron 1994, 50, 12375-12394. 
rationalize the difference in reactivity between azadienes 1a and 1i. 16

AM 1 calculated energies for both HOMO and LUMO of azadiene $\mathbf{l i}$ are shown in Table 6 . The calculated HOMO energy of azadiene $\mathbf{1} \mathbf{i}$ is similar to that of $\mathbf{1 a}$, while the energy of the LUMO is considerably higher.

Then, considering the AM 1 results for the relative frontier orbitals' energies of $\mathbf{1 a}$ and $\mathbf{1 i}$, we should expect azadiene $\mathbf{1 i}$ to exhibit similar reactivity toward electrondeficient dienophiles and to be considerably less reactive toward electron-rich dienophiles when compared with azadiene $\mathbf{1 a}$.

The unsuccessful attempt to promote the cycloaddition of $\mathbf{1 i}$ with enamines is easily explained by the AM 1 calculations which indicate that this azadiene is less activated to participate in the inverse Diels-Alder reaction. In addition to purely electronic effects, steric effects may also play an important role. Indeed the steric effects due to the presence of the two phenyl substituents on C-1 appear to be the main factor of the observed reduced reactivity of $\mathbf{l i}$ toward electron-deficient dienophiles (normal Diels-Alder reaction) compared to $\mathbf{1 a}$, since purely electronic effects due to the substituents were found not to give rise to significant differences in the energy of the HOMO of these two compounds (see Tables 4 and 6). This hypothesis agrees with molecular mechanics data17-19 which indicate that for all the studied compounds but $\mathbf{1 i}$, a low-energy s-cisoide conformation exists, while in this later molecule, steric interaction involving the extra phenyl group and the $\mathrm{CH}_{2}=$ moiety strongly destabilize this form (this molecule was found to adopt exclusively a s-transoide structure).

Reactivity Prediction of Other 2-Azadiene Derivatives. Since azadiene 1c was found to be the most reactive toward electron-rich dienophiles, we decided to estimate the effect on the frontier orbital energies of introducing additional or stronger electron-withdrawing groups on the position 1 of the azadiene, namely adding one extra nitro group to azadiene 1c. AM1 calculations were then undertaken on azadienes $\mathbf{1} \mathbf{j}$ and $\mathbf{1 k}$, bearing dinitrophenyl groups (Table 6). The effect of adding an extra nitro group to azadiene $\mathbf{1 c}$ is to lower both HOMO and LUMO energies. However, the change in the LUMO energy is predicted by the calculations to be more relevant for $\mathbf{1 k}$ than for $\mathbf{1} \mathbf{j}$, and consequently, azadiene $\mathbf{l k}$ is expected to be more reactive in the inverse cycloaddition reaction.

The LUMO-HOMO energy gap associated with the cycloaddition of azadiene $\mathbf{1 k}$ with $\mathrm{N}$-cyclopenten-1-ylpyrrolidine and ethyl vinyl ether is considerably smaller than that associated with the reaction between azadiene $\mathbf{l a}$ and these dienophiles (Table 5). I ndeed, $\Delta \mathrm{E}_{\text {LUмо-номо }}$ for the cycl oaddition reaction involving azadiene $\mathbf{1 k}$ and ethyl vinyl ether is smaller than for the reaction of $\mathbf{l a}$ with the enamine. Azadiene $\mathbf{1} \mathbf{k}$ must then be reactive enough to participate in the Diels-Alder reaction with enol ethers.

AM 1 calculations were also made for azadienes $\mathbf{1 l}$ and Im, bearing a triazine group. Among the azadienes

(17) Molecular mechanics PCFF 300 (101) force fiel $d^{18}$ calculations were undertaken using Cerius $^{2}$ (version 3.5) molecular modeling program. 19

(18) (a) Sun, H. J. Comput. Chem. 1994, 15, 752. (b) Sun, H.; Mumby, S. J .; Maple, J . R.; Hagler, A. T. J . Am. Chem. Soc. 1994, $116,2978$.

(19) Cerius ${ }^{2}$ (version 3.5), Molecular Simulation, San Diego, CA 92111-3712, 1997.
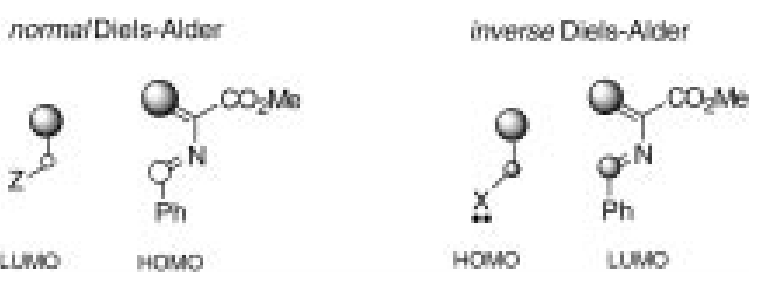

Figure 2. Frontier orbital polarization of electron-deficient (LUMO) and electron-rich (HOMO) dienophiles which are relevant for participation of these species in normal and inverse Diels-Alder reactions, respectively. F avorable polarizations of the diene counterpart frontier orbitals are also shown.

studied, these are the ones which have the lowest calculated HOMO energy and clearly are not able to participate in the normal Diels-Alder reaction. On the other hand, the triazine substituent also lowers considerably the LUMO energy in these two azadienes, activating them for participation in the inverse Diels-Alder reaction.

By comparing the cal culated LUMO energies of $\mathbf{1 l}$ and $\mathbf{1 m}$ with that of $\mathbf{1 c}$, it can be concluded that, while $\mathbf{1}$ is predicted to be less activated than 1c for this type of cycloaddition, $\mathbf{I m}$ is estimated to be slightly more activated than this later. However, the reactivity enhancement going from $\mathbf{1 c}$ to $\mathbf{1 m}$ is certainly not very significant in practical terms.

The effect of adding a halogen to the phenyl group of

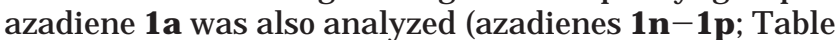
6). The AM 1 calculations predict lower values for HOMO and LUMO energies upon substitution, but the substituent effects are less pronounced than those attained by addition of a nitro group.

In summary, it can be concluded from the whole set of AM 1 calculations carried out for azadienes $\mathbf{1 j}-\mathbf{1 p}$ that azadiene $\mathbf{1} \mathbf{k}$ appears to be the best candidate to start a series of experiments which might allow broadening of the scope of this type of Diels-Alder reaction. Such experimental work is now in progress in our laboratory.

Regioselectivity. Azadiene $\mathbf{l a}$ shows high regioselectivity in the reaction with both methyl vinyl ketone (Table 1) and N-cyclohex-1-enyl pyrrolidine (Table 2). The polarization of the relevant frontier orbital for participation in Diels-Alder reactions (normal and inverse type, respectively) of electron-rich and electron-deficient dienophiles has been shown to be as schematically drawn in Figure 2.20

The AM 1 calculated polarization of the relevant frontier orbitals of azadiene la shows a higher contour value at C-4 than at C-1 in both LUMO and HOMO orbitals (Figure 3). This polarization explains the observed regioselectivity in the normal and inverse Diels-Alder reactions of this compound. On the other hand, the calculations predict that both orbitals are nearly symmetrical with respect to the $\mathrm{C}=\mathrm{N}-\mathrm{C}=\mathrm{C}$ plane, a result which is in agreement with the fact that no endo/exo selectivity has been experimentally observed. ${ }^{2}$

Barluenga et al. ${ }^{21}$ have studied the reactivity of the electron-deficient acyclic azadiene $\mathbf{1 q}$ with enamines, and the regioselectivity is the same shown in the cycloaddition reactions of azadiene $\mathbf{1 a}$ (Scheme $\mathbf{1}$ ).

(20) Houk, K. N. J . Am. Chem. Soc. 1973, 95, 4092-4094.

(21) Barluenga, J .; Tomás, M.; Ballesteros, A.; Gotor, V. J . Chem. Soc., Chem. Commun. 1987, 1195-1196. 


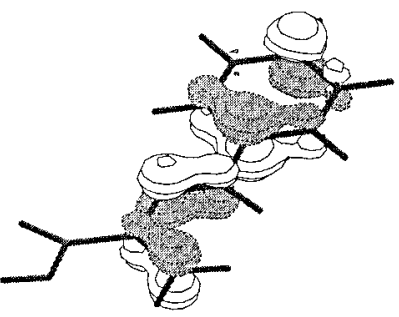

a)

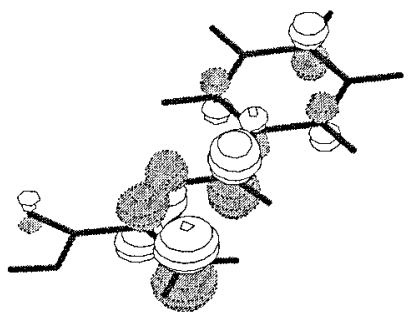

b)
Figure 3. AM1 calculated polarization of the HOMO (a) and LUMO (b) orbitals of azadiene $\mathbf{1 a .}$

\section{Scheme 1}

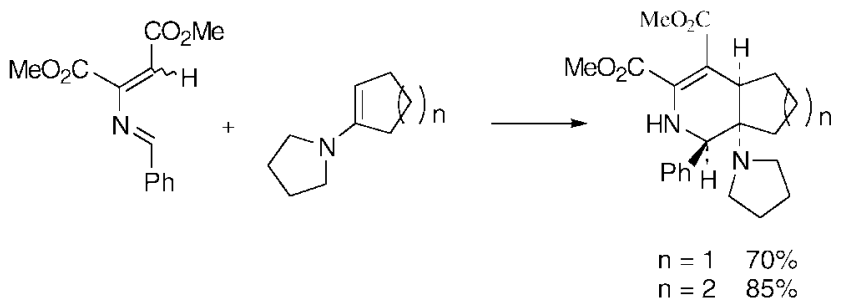

Scheme 2

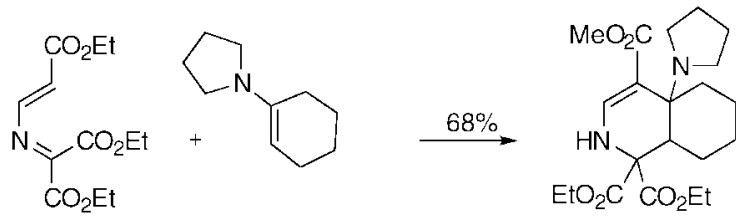

However, Palacios et al. ${ }^{22}$ have studied the cycloaddition reaction of azadiene $\mathbf{I r}$ with the same kind of dienophiles and shown that it presents a different regioselectivity (Scheme 2).

The AM 1 calculations undertaken on these two azadienes indicated similar values for their LUMO energy levels. Consequently, the same reactivity should be expected for the inverse Diels-Alder reaction of these compounds. Comparison of the AM 1 calculated LUMO energies for azadienes $\mathbf{1 q}$ and $\mathbf{1 r}$ and for azadiene $\mathbf{1 c}$ (see Tables 3 and 7) leads to the conclusion that these compounds shall be slightly less reactive than 1c. Indeed, in agreement with the results of the AM 1 calculations, for $\mathbf{1 q}$ and $\mathbf{1 r}$ the cycloaddition was found to be efficient only when very activated dienophiles are used. ${ }^{22}$

The calculated polarization of the HOMO of azadiene 1q indicates that C-4 is slightly more nucleophilic than

(22) Palacios, F .; Heredia, I. P.; Rubiales, G. J . Org. Chem. 1995, 60, 2384-2390.
C-1, while in the case of $\mathbf{1 r}$ the contour values around these atoms are similar. On the other hand, for both molecules the LUMO is slightly more located at C-4 than at $\mathrm{C}-\mathbf{1}$. Hence, the polarization of the LUMO in both $\mathbf{1 q}$ and $\mathbf{l} \boldsymbol{r}$ is the same as in azadiene $\mathbf{l a}$, though the predicted degree of polarization is considerably reduced in these compounds. In the case of $\mathbf{1 q}$, where steric effects do not seem to play an important role, the regioselectivity is essentially determined by purely electronic effects and, thus, it is the same as for $\mathbf{1 a}$. On the other hand, since azadiene $\mathbf{l r}$ has two substituents at C-1, steric effects become dominant and the preferred regiosel ectivity is the one where the less-hindered carbon of the enamine attaches to $\mathrm{C}-1$ of the azadiene.

All the above observations indicate that the regioselectivity of 2-azadienes is strongly determined by the nature of the substituents.

\section{Conclusion}

The unusual reactivity of azadiene $\mathbf{1 a}$, characterized by the participation in both the normal and inverse Diels-Alder reactions, can be attributed to the fact that HOMO and LUMO energy levels are rather close. AM 1 calculations of the energies of the frontier orbitals of azadienes $\mathbf{1 b}$ - $\mathbf{1 f}$ show that among the studied molecules azadiene $\mathbf{l c}$ is the one having the lower-energy value for the LUMO orbital. This prediction agrees with the experimental findings where azadiene $\mathbf{1 c}$ has been shown to be the most reactive toward electron-rich dienophiles. AM1 predictions of the reactivity of other 2-azadiene derivatives ( $\mathbf{1} \mathbf{j}-\mathbf{1 p})$ were also made, enabling the conclusion that methyl 1-(2,4-dinitrophenyl)-2-aza-1,3-butadiene (1k) might al low the broadening of the scope of the inverse type Diels-Alder reaction.

The orbital polarization predicted for azadiene la shows that both HOMO and LUMO are more localized on C-4 than on C-1, thus being in agreement with the experimentally observed regioselectivity of this azadiene in the inverse and normal Diels-Alder reactions. The polarization of the frontier orbitals was also evaluated for azadienes $\mathbf{~} \mathbf{q}$ and $\mathbf{l r}$ and, together with steric considerations, could also be used to explain successfully the different regioselectivity observed for these compounds.

Acknowledgment. We thank Chymiotechnon and Projecto Praxis XXI no. 2/2.1/QUI/390/94 for financial support.

J O980090E 